\title{
Utilizing Scanning Transmission Electron Microscopy to Locate and Image Raman Active Gold Core Nanoparticles in Mouse Tissue
}

\author{
P.J. Kempen*, A.S. Thakor**, C.L. Zavaleta, S.S. Gambhir**, and R. Sinclair* \\ * Department of Materials Science and Engineering, Stanford University, 496 Lomita Mall, Stanford, \\ CA 94305-4034 \\ **Molecular Imaging Program at Stanford, Departments of Radiology \& Bioengineering, Stanford \\ University, Stanford CA 94305
}

Raman active gold core nanoparticles (R-AuNPs), which we use for surface-enhanced Raman scattering (SERS), have a $60 \mathrm{~nm}$ diameter gold core and $30 \mathrm{~nm}$ thick silica shell. An organic Raman layer is adsorbed onto the gold core. The R-AuNPs are produced by Oxonica Inc and provide a surface enhancement effect resulting in an amplified Raman signal that can be used to track them in a biological environment[1,2].

We developed a method to utilize scanning transmission electron microscopy (STEM) to locate and image these nanoparticles in tissue after intravenous injection in living mice. STEM was chosen because it offers a number of benefits over fixed beam transmission electron microscopy for locating gold-silica core-shell nanoparticles in mouse tissue. In STEM it is possible to achieve very high scan rates by decreasing the dwell time to $0.8 \mu \mathrm{s} /$ pixel. Due to the high atomic number of gold there is still enough mass contrast in the bright field image to indentify the R-AuNPs. This allows for the examination of "large" volumes of tissue in relatively short periods of time. In addition, the ability to set the dynamic range of the bright field STEM detector allows us to visually differentiate between gold nanoparticles and the various salts that may form during fixing, staining, and dehydration. Finally, decent mass contrast is still obtained at very small spot sizes, resulting in a much more stable sample than would otherwise be achieved at comparable mass contrast in TEM.

The R-AuNPs were intravenously injected into the mouse through the tail vein at a dose of $9.6 \mathrm{x}$ $10^{10}$ nanoparticles. After 24 hours the mouse was sacrificed and the liver was fixed, stained and dehydrated utilizing standard procedures[3]. $150 \mathrm{~nm}$ sections were cut using a Leica Ultracut $\mathrm{S}$ microtome and placed on 200 mesh bare copper grids. The samples were examined using a Tecnai G2 F20 X-Twin TEM operating at $120 \mathrm{kV}$, with gun lens equal to 6 and spot size equal to 10 in terms of standard Tecnai settings. 340 gold-silica core-shell nanoparticles were located in 12150 $\mu \mathrm{m}^{3}$ of material. This indicates that the liver is filtering these nanoparticles out of the blood as it should. The majority of these nanoparticles were located in clusters, with the large variation in the number of nanoparticles per cluster. These clusters were predominantly located inside vesicles in the tissue as shown in Figure 1. Another interesting feature of these images is the presence of the silica shell around the gold core. In TEM this silica shell is rarely visible, most likely due to the similarities in atomic number with the surrounding biological matrix, however in STEM the silica shell is always visible surrounding the gold core as shown in Figure 2.

STEM provides a better method than TEM to analyze gold nanoparticles in tissue samples. The ability to examine "large" volumes of tissue while maintaining high contrast ratios combined with the enhanced ability to image the nanoparticle structure, including simultaneous bright field and dark field imaging, demonstrate why STEM is superior to TEM for imaging this system. [4] 
[1] C. Zavaleta et al. PNAS. 105(15) (2008), 5844-5849.

[2] C. Zavaleta et al. PNAS 109(32) (2009), 13511-13516.

[3] A.L. Koh et al. Ultramicroscopy 109 (2008), 111-121.

[4] This research is supported by the Center for Cancer Nanotechnology Excellence Focused on

Therapy Response (CCNE-TR) grant NCI CCNE U54 CA119367 (SSG)

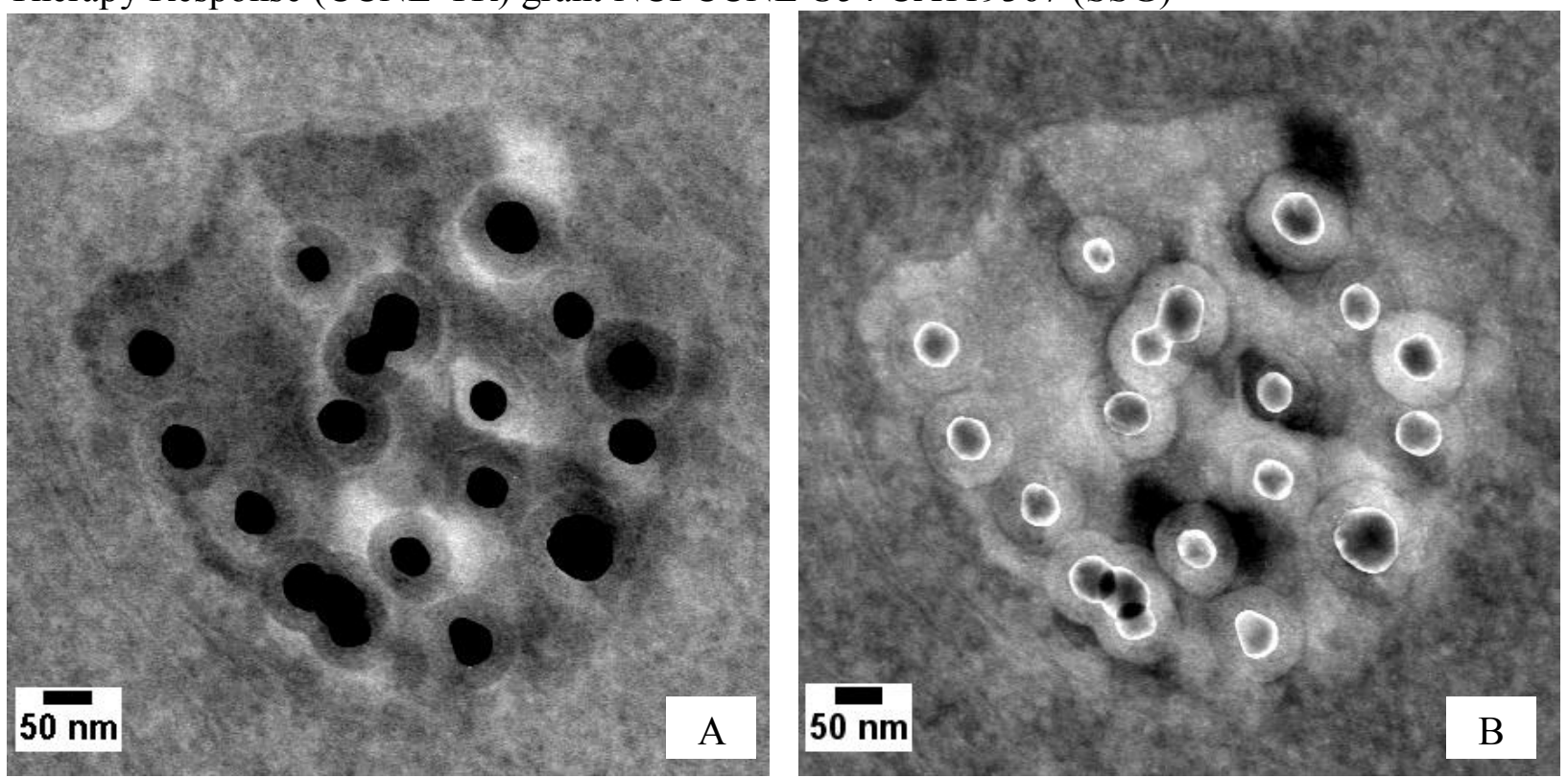

Fig 1. (A) Bright field STEM and (B) dark field STEM images taken at $120 \mathrm{kV}$ showing the clusters of gold-silica core-shell nanoparticles inside a vesicle in the mouse liver tissue sample.

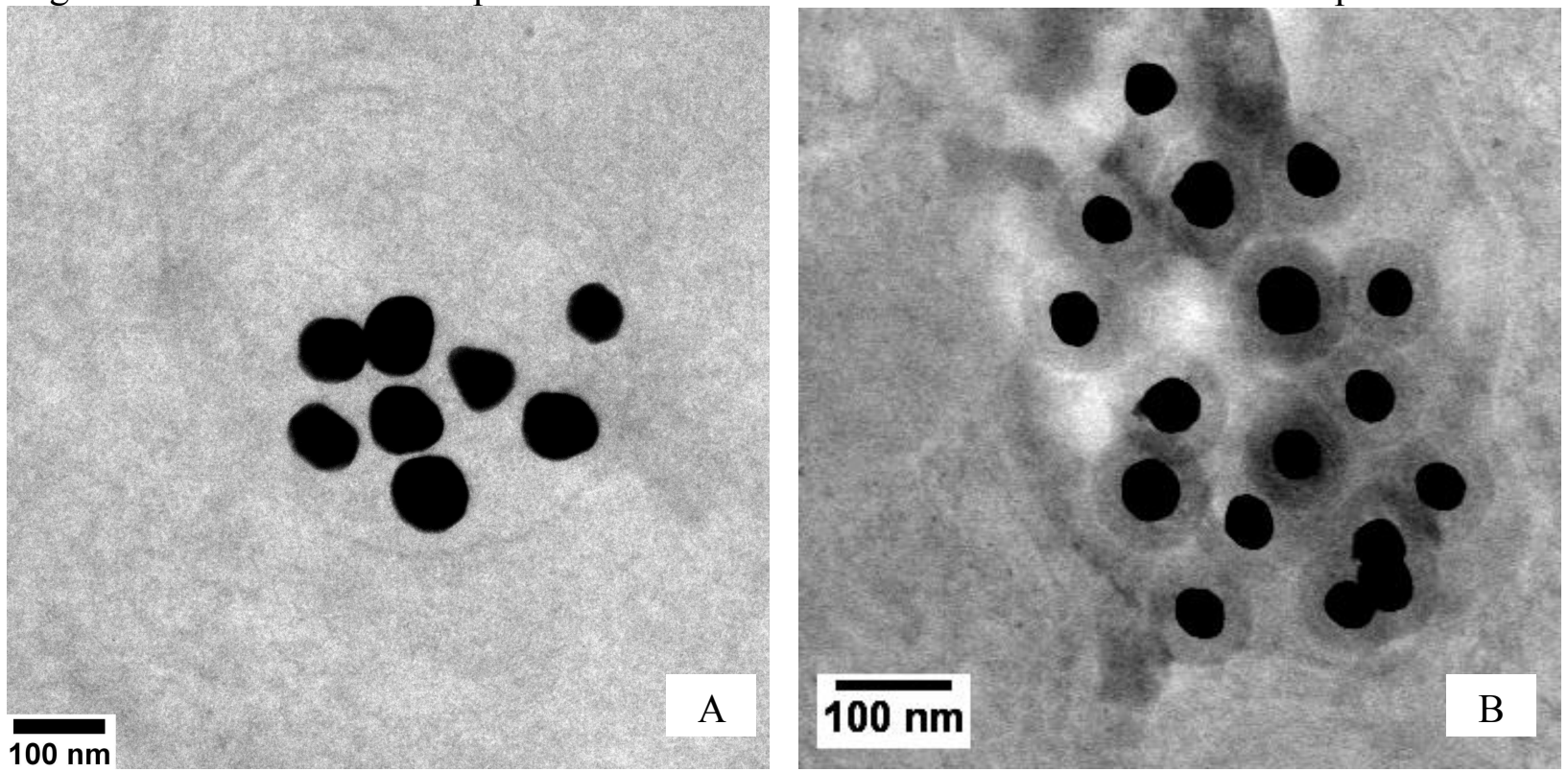

Fig 2. (A) Bright field TEM image of gold-silica core-shell nanoparticles in a HeLa cell taken at 120 $\mathrm{kV}$ showing that the silica shells of the core-shell nanoparticles are not visible. (B) Bright field STEM image of gold-silica core-shell nanoparticles in mouse liver tissue taken at $120 \mathrm{kV}$ showing the presence of the silica shell on the gold-silica core-shell nanoparticles. 